\title{
Catastrophic Rupture Induced Damage Coalescence in Heterogeneous Brittle Media
}

\author{
Feng Rong, ${ }^{1}$ Haiying Wang, ${ }^{1}$ Mengfen Xia, ${ }^{1,2}$ Fujiu Ke, ${ }^{1,3}$ and Yilong Bai ${ }^{1}$
}

\begin{abstract}
In heterogeneous brittle media, the evolution of damage is strongly influenced by the multiscale coupling effect. To better understand this effect, we perform a detailed investigation of the damage evolution, with particular attention focused on the catastrophe transition. We use an adaptive multiscale finite-element model (MFEM) to simulate the damage evolution and the catastrophic failure of heterogeneous brittle media. Both plane stress and plane strain cases are investigated for a heterogeneous medium whose initial shear strength follows the Weibull distribution. Damage is induced through the application of the Coulomb failure criterion to each element, and the element mesh is refined where the failure criterion is met. We found that as damage accumulates, there is a stronger and stronger nonlinear increase in stress and the stress redistribution distance. The coupling of the dynamic stress redistribution and the heterogeneity at different scales result in an inverse cascade of damage cluster size, which represents rapid coalescence of damage at the catastrophe transition.
\end{abstract}

Key words: Heterogeneity, damage evolution, FEM, damage coalescence, catastrophic rupture, stress redistribution.

\section{Introduction}

Damage evolution and rupture of disordered heterogeneous brittle media, such as rocks and the earth's crust, is a key problem in science, especially in earthquake simulations (RUNDle et al., 2000; BEN Zion and SAMmis, 2003; MORA et al., 2002). The catastrophe transition occurring in such media is the most important feature in this problem (BAI et al., 1994; XIA et al., 2002; WEI et al., 2000). In rock mechanics (JAEGER and COOK, 1979), it is found that at the beginning of loading, micro-damages appear on mesoscopic heterogeneities. With increasing load, some disordered weakness at mesoscales may be amplified strongly due to strong and trans-scale stress redistribution, and become significant

\footnotetext{
${ }^{1}$ State Key Laboratory of Nonlinear Mechanics (LNM), Institute of Mechanics, Chinese Academy of Sciences, Beijing 100080, China. E-mail: rongf@hotmail.com

${ }^{2}$ Department of Physics, Peking University, Beijing 100871, China.

${ }^{3}$ Department of Applied Physics, Beihang University, Beijing 100083, China.
} 
effects on the global scale. Beyond the catastrophe point, the responses of the sample, such as damage patterns, energy release, etc., change dramatically with increasing controlling variable. In other words, the system displays trans-scale catastrophic transition.

Though material heterogeneity has been examined in a number of models, such as fiber bundle model (DANIELs, 1945; COLEMAN, 1958; CURTIN, 1997), coupled pattern mapping model (XIA et al., 2000; WEI et al., 2000), network model etc., the mechanisms underlying the catastrophic transition through various spatial scales have not been clearly revealed in computational simulations (CAO et al., 2002). Therefore, it is of the deepest interest to reveal the characteristic features in the catastrophe transition and to seek its underlying mechanism.

Recent works in the study of multiscale coupling (BAZANT and CHEN, 1997; WANG et al., 2004; RoNG et al., 2006) suggest that damage evolution and rupture of solid can be regarded as a trans-scale process in a wide range of spatiotemporal scales. Traditionally, researchers working on computational simulations concentrated on bringing practical details into their models, but ignored various spatial scales inherent in heterogeneous media. To obtain proper resolution of small-scale damage effects, finite-element meshes consisting of several million elements are necessary. These often require hours of computation time on even the latest supercomputer and make the analysis of multiscale damage evolution almost impossible. This severely limits the usefulness of computation. Although there are many previous works on the multiscale numerical scheme, such as multilevel method (FISH and CHEN, 2003) and adaptive VCFEM (GHOSH et al., 2001), most of them use the asymptotic homogenization and can be quite effective in analyzing the scale effect, especially in the debonding or crack evolution. However, our concern is the multiscale effects of damage evolution induced catastrophe of heterogeneous media. The detailed stress fields are not enough and the coupling among the micro-damages should be considered to cope with these effects (KRAUSE and RANK, 2003). Therefore, it is necessary to develop a numerical model, which can effectively deal with the coupling of medium heterogeneity and dynamic stress redistribution.

On the other hand, as noted previously that in heterogeneous brittle media, the micro-damages are usually initiated from small scale at some weak locations. Then, micro-damages develop from small to large scales via growth and coalescence, and eventually trigger catastrophe transition. In order to simulate such a phenomena, we must turn to the adaptive scheme. However, in the standard adaptive finite-element algorithm, its constitutive relation most often keeps the same in all meshes (regardless of how large or small), hence it cannot depict heterogeneity properly (Rong et al., 2005b).

In this paper, a Multiscale adaptive Finite-Element Model (MFEM) is proposed, and some 2-D problems, both plane stress and plane strain cases, are simulated with the MFEM. Based on the numerical results, it is found that damage 
coalescence from mesoscopic to macroscopic scales is crucial in the catastrophe transition. In particular, the underlying mechanism of catastrophe transition, namely the coupling between dynamic stress redistribution and disordered heterogeneity on multiscales, is exposed based on the analysis of the numerical results.

\section{Adaptive Multiscale FEM (MFEM)}

Since its origin in the early 1960s, FEM is perhaps the most widely applied numerical method across the science and engineering fields (ZIENKIEWICZ and TAYlOR, 1988; JING and HUDSON, 2002). The proposed MFEM is actually a multilevel and self-adaptive one based on the traditional finite-element model. As damage accumulates, the dangerous element will be adaptively refined to a smaller scale. The 4-8 variable nodes serendipity element (KIKUCHI et al., 1999) is adopted to connect a two-scale effect and the polynomial interpolation functions used in the model follow $C^{0}$ continuity (SzABO and BABUSKA, 1991).

\subsection{Constitutive Relationship and Damage Criterion}

For simplification, a linear elastic constitutive relation is used for intact elements, i.e.,

$$
\varepsilon=E \sigma
$$

where $\boldsymbol{E}$ is the modulus matrix of elements, $\boldsymbol{\varepsilon}$ and $\boldsymbol{\sigma}$ are the strain and stress tensors, respectively. For damage occurrence, Coulomb criterion is adopted for brittle crack (JAEGAR and COOK, 1979):

$$
\tau_{M C}=|\tau|-\mu \sigma \geq \tau_{s},
$$

where $\tau_{M C}$ is the Coulomb stress of an element, $\mu$ is the frictional coefficient, $\tau$ and $\sigma$ are the shear and normal stresses (compressive stress is defined to be positive) of the element respectively, and $\tau_{S}$ is the inherent shear strength. The physical meaning of this inequality is as follows. If $\tau_{M C}<\tau_{S}$ for a particular element, it will remain intact. Otherwise, it will be refined into four smaller elements. If the criterion is also satisfied in the smaller element resulting from the mesh refinement, the smaller element will be further refined into four much smaller elements. This adaptive mesh refinement will continue until the finest elements. The finest element still has $\tau_{M C} \geq \tau_{S}$, thus, it will be considered damaged and can no longer support shear stress on the corresponding direction.

The material heterogeneity is introduced by Weibull distribution of $\tau_{S}$ :

$$
h\left(\tau_{S}\right)=m\left(\frac{\tau_{S}^{m-1}}{\eta^{m}}\right) \exp \left[-\left(\frac{\tau_{S}}{\eta}\right)^{m}\right],
$$


where $m$ and $\eta$ are the Weibull modulus and scale parameter, respectively. Therefore, each finest element has a given $\tau_{s}$, according to Equation (2). On the other hand, corresponding spatial distribution in a specimen is assumed to be random. Moreover, for a coarse element, $\tau_{s}$ is supposed to be the average of four smaller elements with $\tau_{s}$ in it. The frictional coefficient $\mu$ and the scale parameter $\eta$ are assumed identical for all elements in the simulations.

Actually, Equation (2) can be satisfied on three different directions within an element (Fig. 1a). In order to simplify the simulation, the response of an element beyond the criterion of (2) is assumed to follow a modified smeared crack model (BAZANT and CHen, 1997; Rong et al., 2005b) to simulate the post-damage effect. That is, if an element has become damaged and the stress normal to the damage is tension, the failed element is described by a reduced elastic modulus $E$ (Equation (4), $R$ is taken to be $10^{3}$ in the simulation). However, if the normal stress is compressive, i.e., $\sigma_{n}$ is positive, here $n$ is the normal to damage, see Figure 1(a), the elastic modulus will remain to be the initial one $E_{0}$ (Equation 4).

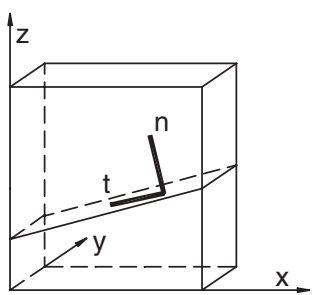

(1)

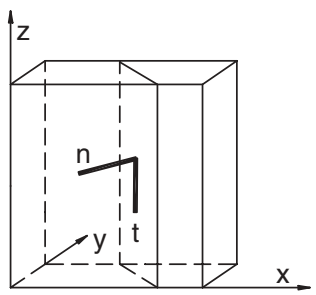

(2)

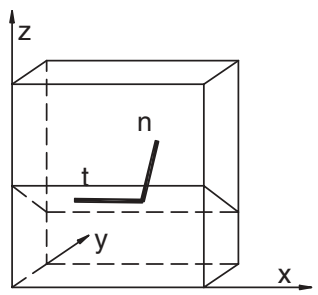

(3)

(a)

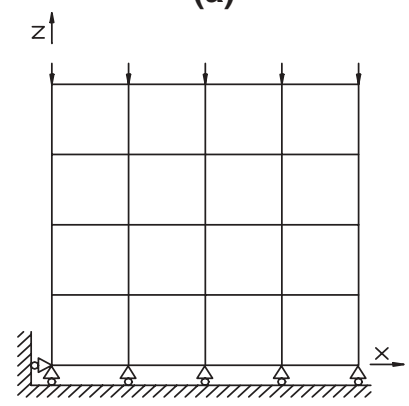

(b)

Figure 1

(a) Possible three-dimensional orientation of damage in elements, $n$ and $t$ is the normal direction and the tangential direction of damage direction, respectively. (b) Simplified diagram of the FEM model, indicating boundary conditions. 


$$
E= \begin{cases}E_{0} / R & \sigma_{n}<0 \\ E_{0} & \sigma_{n} \geq 0\end{cases}
$$

where $E$ is the Young's modulus in modulus matrix of elements, $E_{0}$ is the initial Young's modulus, $R$ is the reduction ratio and $\sigma_{n}$ is the stress normal to the damage. Poisson's ratio in the damaged elements is assumed to be identical to the intact one in the simulation. Hence there might be three different damage states in an element and each one will result in a reduction of Young's modulus in corresponding damage directions. If an element cracked on a direction, the damage fraction (defined on the element) is assumed to increase $1 / 3$, and only the element has cracks on all three orientations. The mechanical response of the element will be depicted with a total reduced stiffness matrix. Correspondingly, the element should be regarded as a partially damaged element or fully damaged one depending on how many directions are damaged. More details of the discussion on various partially damaged states are available in RoNG et al. (2005). In fact, experimental results (PATERSON, 1978) of uniaxial stress in rock mechanics demonstrate that the main crack (plane of failure) occurs on a plane that is inclined to the loading direction. This corresponds to orientations shown in Figure 1a(1) or (3).

The system of Equations (1-4) forms the basic constitutive formation of MFEM in the simulation.

\subsection{Case Studies with MFEM}

As described in Section 2.1, we examine two-dimensional (2-D) cases: plane stress and plane strain. Note that although the problems are 2-D, damage can form in three different directions according to the nature of the Coulomb criterion.

The boundary conditions used in the simulations are as follows: compressive axial load is acted on upper-surface while the lower surface is fixed, as shown in Figure 1b. The controlling variable in calculations is the upper-surface boundary displacement, namely the displacement increment is applied step by step on the upper surface until the failure of the sample. By solving minimum energy, we let force field reach equilibrium. Note that this equilibrium state of force field does not mean the equilibrium of damage due to the stress redistribution. Thus in one step, the load is held until no more elements become damaged due to stress redistribution.

Hence, in the simulation, the quasi-static loading mode is used. This implies

$$
t_{P} \ll t_{\mathrm{D}} \ll t_{\mathrm{ex}}
$$

where $t_{P}$ is the characteristic time for stress to reach equilibrium, $t_{\mathrm{D}}$ is the characteristic time for damage to nucleate and $t_{\mathrm{ex}}$ is the characteristic external loading time. The relationship in (5) implies that the stress field will equilibrate much faster than damage forms. Additionally, damage nucleates faster than the change of 
Figure 3

Damage evolution patterns of lane stress case. The labels (A-E, also marked on the $\sigma-\varepsilon$ curve) denote the quasi-static loading steps 2, 202, 502, 531 and 532, respectively. In the patterns, there are three kinds of darkness; black indicates fully damaged, gray indicates partially damaged, and white is still intact.

the external force. These effectively reduce our problems to be a quasi-static one. Therefore, the simulation is effectively a time series of FEM solutions to the elastostatic problem.

Additionally, as a case study, the following parameters (JAEGER and COOK, 1979 and XU et al., 2004) are used in the simulations:

Young's modulus $E_{0}=47.2 \times 10^{9} \mathrm{~Pa}$,

Poisson Ratio $v=0.250$,

Frictional coefficient $\mu=0.639$,

Weibull modulus and scale parameter $m=3$ and $\eta=3.10 \times 10^{5} \mathrm{~Pa}$.

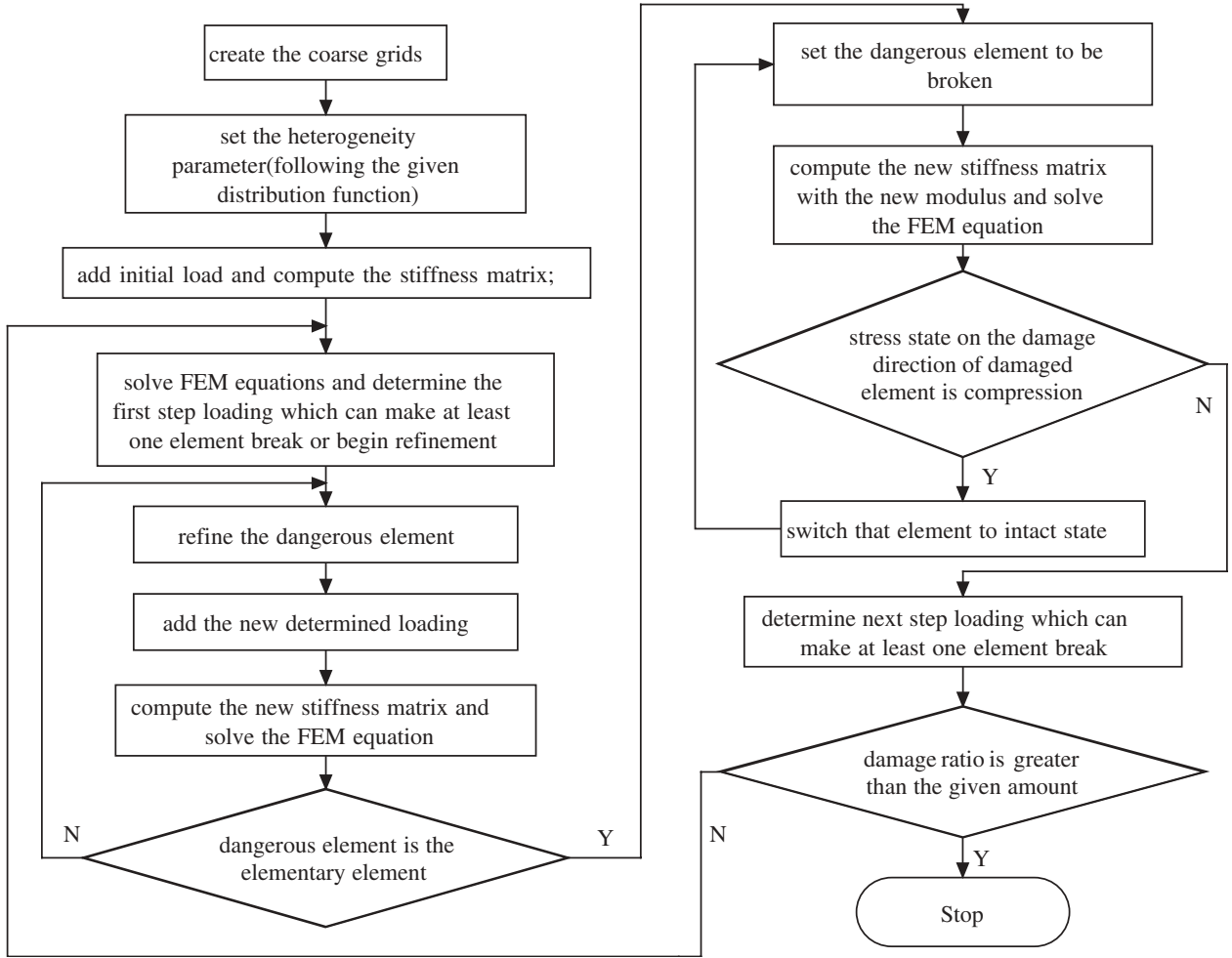

Figure 2

Flow chart of MFEM. 

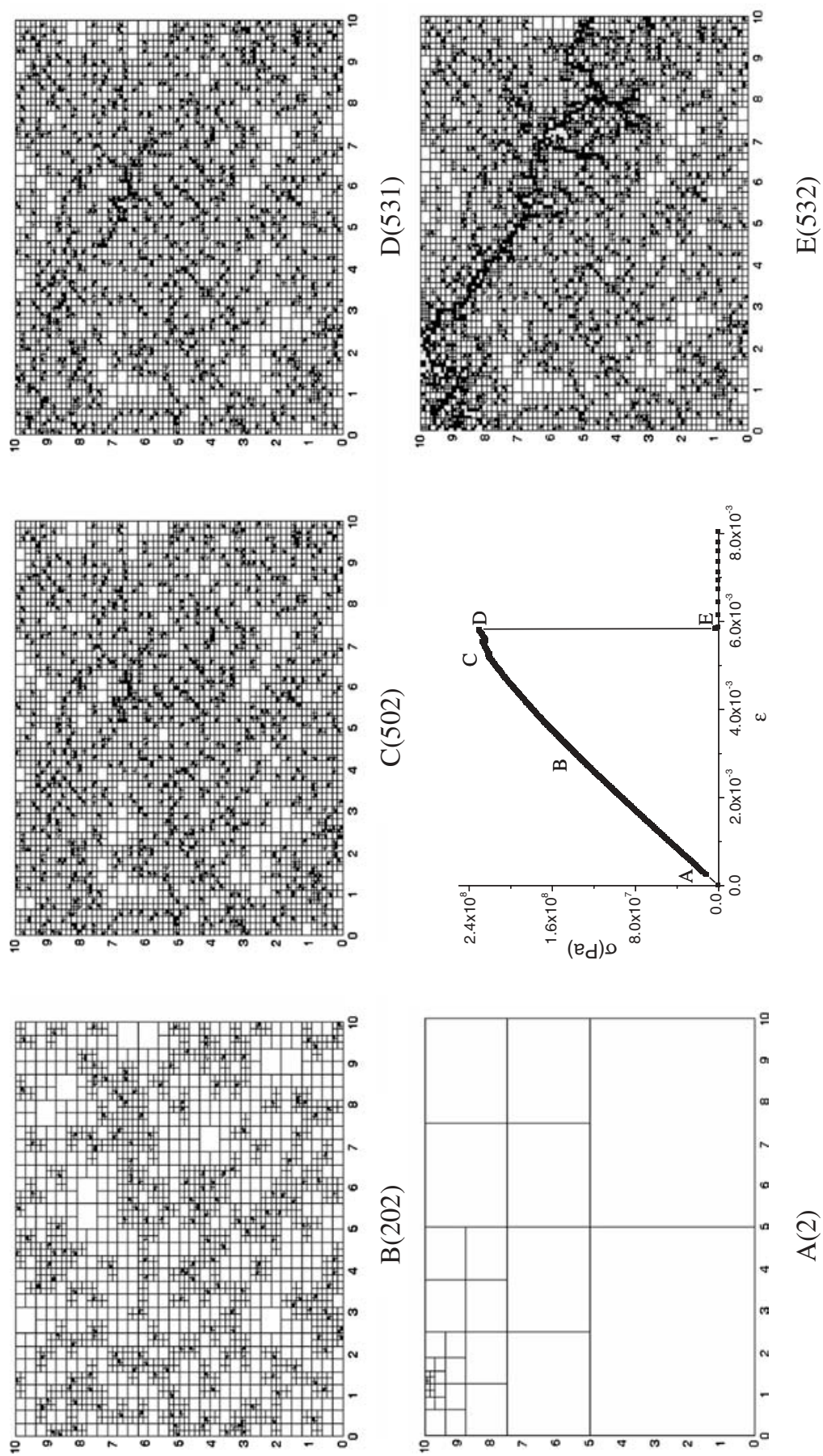
Figure 4

Damage evolution patterns of plane strain case. The labels (A-E, also marked on the $\sigma-\varepsilon$ curve) denote the quasi-static loading steps 2, 250, 500, 648 and 649, respectively. In the patterns, for each square element, there are three kinds of darkness; black indicates fully damaged element, gray indicates partially damaged, and white still remains intact.

In the end, for the sake of clarification of the algorithm, the flow chart of MFEM is presented in Figure 2.

\section{Damage Evolution and Catastrophe Transition}

We examined 2-D problems of plane stress and plane strain using the MFEM. To simulate initial heterogeneity, 15 different spatially random distributions of material strength, following a Weibull distribution, are given over the FEM finest scale elements. This produces different patterns of the inherent shear strength while maintaining the identical probability distribution among the 15 samples. We use the results from both plane stress and plane strain cases to analyze the physical processes that govern the catastrophe transition.

All 15 samples demonstrate a similar evolution trend, namely nonlinear deformation with random damage at an early stage and an abrupt catastrophe transition with trans-scale coalescence. A representative damage evolution pattern for the plane stress case is shown in Figure 3. The central diagram is the curve of nominal stress versus nominal strain in the entire process. At the initial stage, damage appears to be relatively uniform (step 202). The damage occurred at the elements with the lower shear strength in the strength distribution; see Figure 3B and we will discuss this issue in detail later. With increasing loading, more and more elements satisfy Equation (2) and become damaged. This is called damage accumulation; see B and $\mathrm{C}$ in Figure 3. Then, more damage clusters are formed (C and D in Fig. 3) but the whole sample still remains stable. Finally, a catastrophic rupture happens at step 532 (from D to E in Fig. 3).

Similarly, a typical damage evolution pattern for plane strain is shown in Figure 4. Although the detail of heterogeneity in this case is identical to that of the previous plane stress case, the final spatial damage pattern is quite different (compare Figs. $3 \mathrm{E}$ and $4 \mathrm{E}$ ). The mean of catastrophe strain in 15 plane stress cases is $6.04 \times 10^{-3}$, while that of 15 plane strain cases is $7.95 \times 10^{-3}$. The comparison shows that the strain at the catastrophe transition for the plane strain case seems to be greater than that of plane stress, although these two have the identical initial spatial pattern of the inherent shear strength.

Hence, understanding the factors that contribute to these differences is an important part of understanding the catastrophe transition and the process of material failure. 

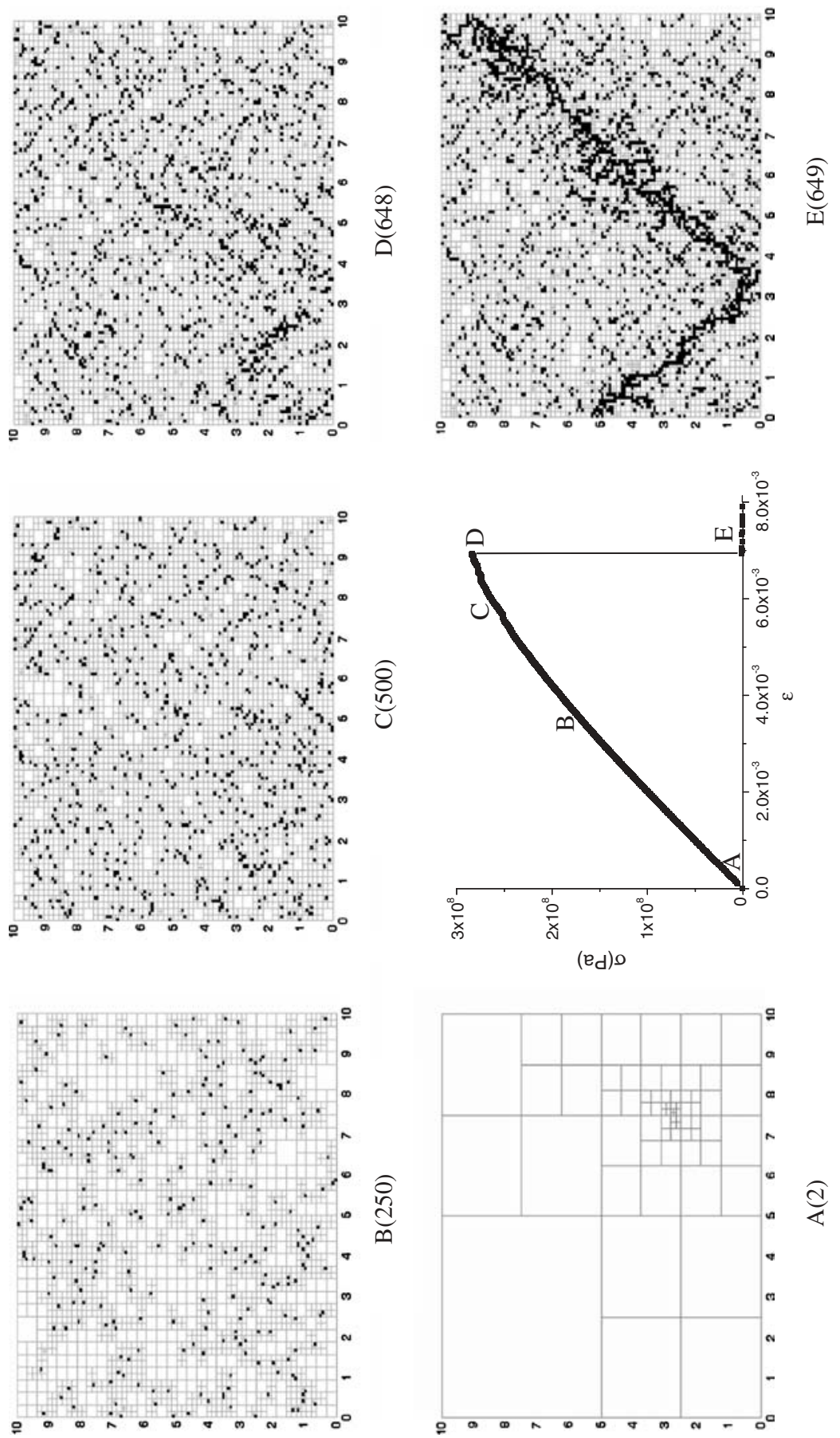


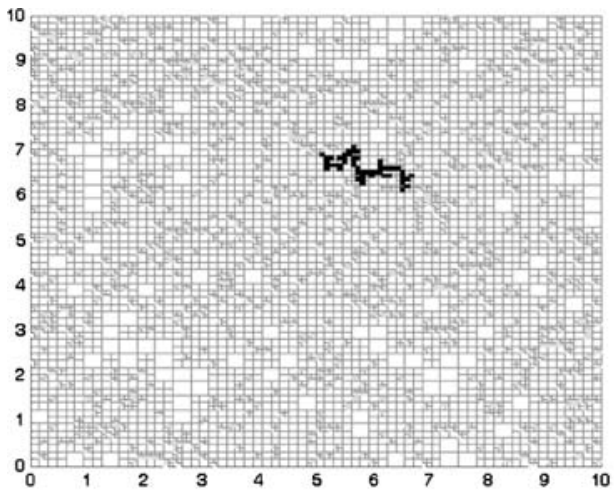

(a) Substep 1

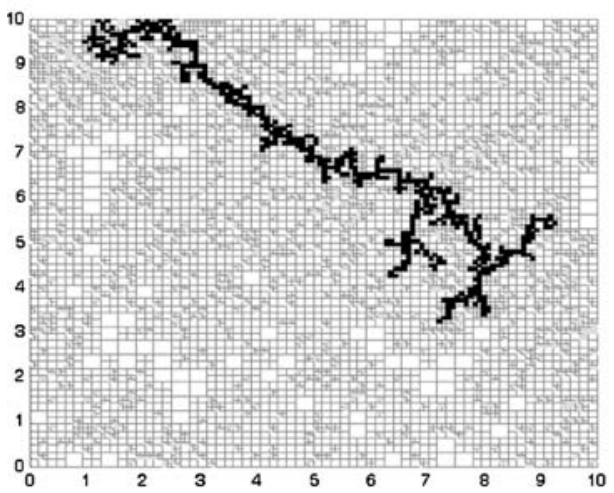

(c) Substep 27

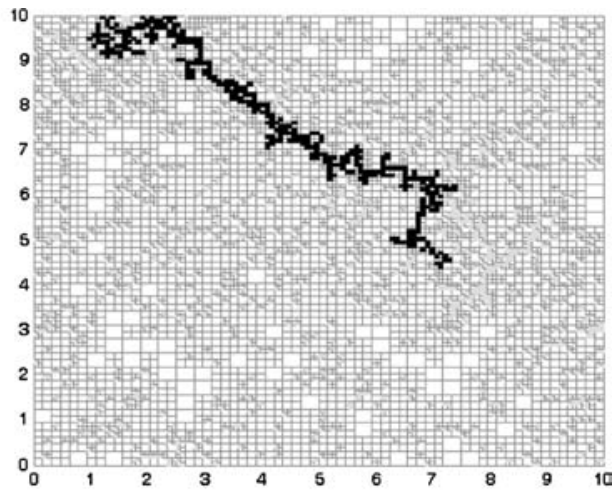

(b) Substep 26

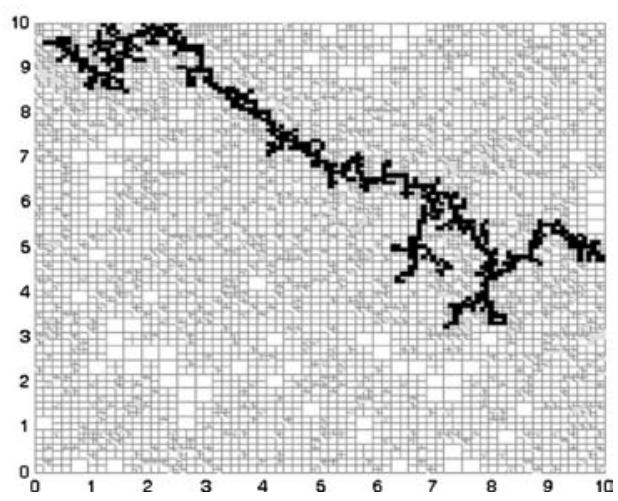

(d) Substep 33

Figure 5

The damage coalescence of the plane stress case during the catastrophic transition. In the pattern there are three kinds of elements with different chroma; black indicates the damaged elements forming the largest damage cluster, gray indicates damaged elements elsewhere, and white elements are still intact.

\section{Trans-scale Damage Coalescence}

It has been suggested that catastrophic rupture can mainly be attributed to trans-scale damage coalescence from mesoscopic to macroscopic, and eventually, to global scales (WANG et al., 2004). Figure 5 shows the nonequilibrium formation of the largest damage cluster during the catastrophic rupture in the plane stress case, i.e., from D (step 531) to E (step 532) in Figure 3. As described before, during stable damage accumulation, both stress and damage reach equilibrium in each step. However, during this catastrophic transition, we should calculate a number of substeps to reveal the trans-scale coalescence. The so-called substeps stand for the procedure, in which even though stress reaches equilibrium, damage still evolves 


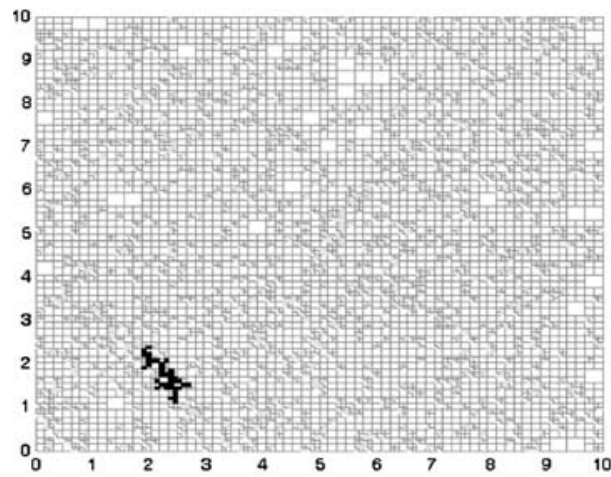

(a) Substep 1

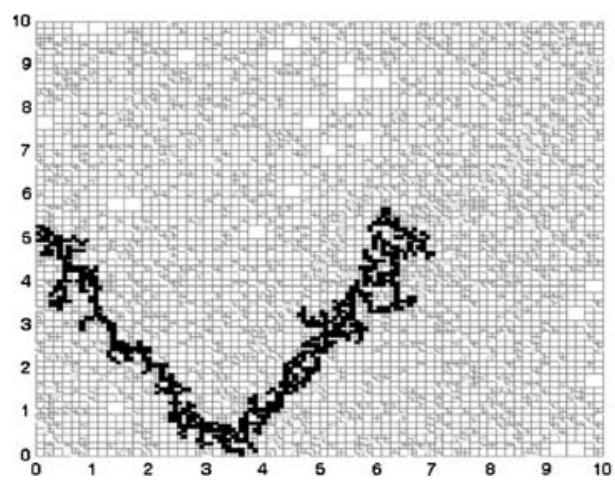

(c) Substep 27

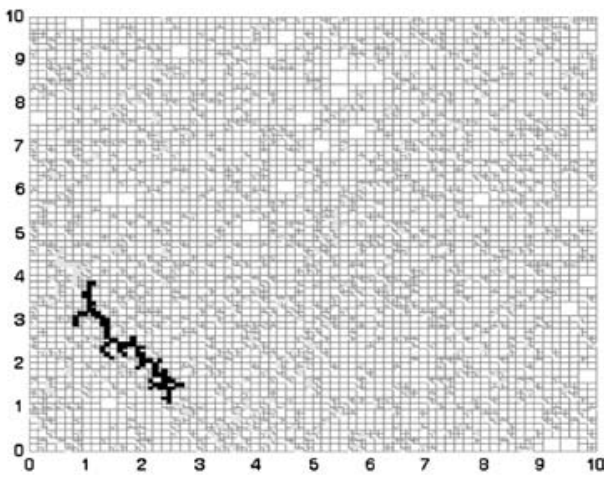

(b) Substep 26

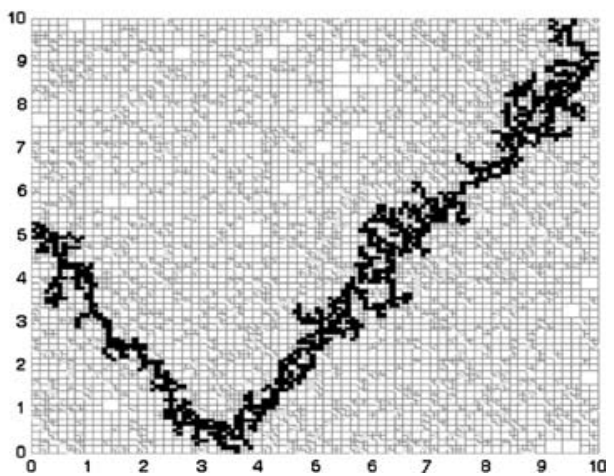

(d) Substep 33

Figure 6

The damage coalescence of the plane strain case during the catastrophic transition. In the pattern, there are three kinds of elements with different chroma; black indicates the damaged elements forming the largest damage cluster, gray indicates damaged elements elsewhere, and white elements are still intact.

and violates the current equilibrium stress state, and then continue on until complete failure. Figure 5 shows the trans-scale damage coalescence of collectively 33 substeps during this non equilibrium catastrophic transition. Moreover, the growth of the cluster in the catastrophe transition is far different from a crack propagation, namely the largest cluster is sometimes formed with the connection of two neighboring ones (for example see (b) and (c) in Figure 5).

Similarly, the nonequilibrium formation of the largest damage cluster during the catastrophic rupture in the plane strain case is given in Figure 6. The growth of the largest damage cluster in the plane stress and plane strain cases is shown in Figures 7 and 8 , respectively. In these two figures (7 and 8), we define the characteristic ratio as the ratio between the widths of the damage cluster to the width of the sample. When the characteristic ratio becomes greater than 0.1 , we can 
(c) Step 532

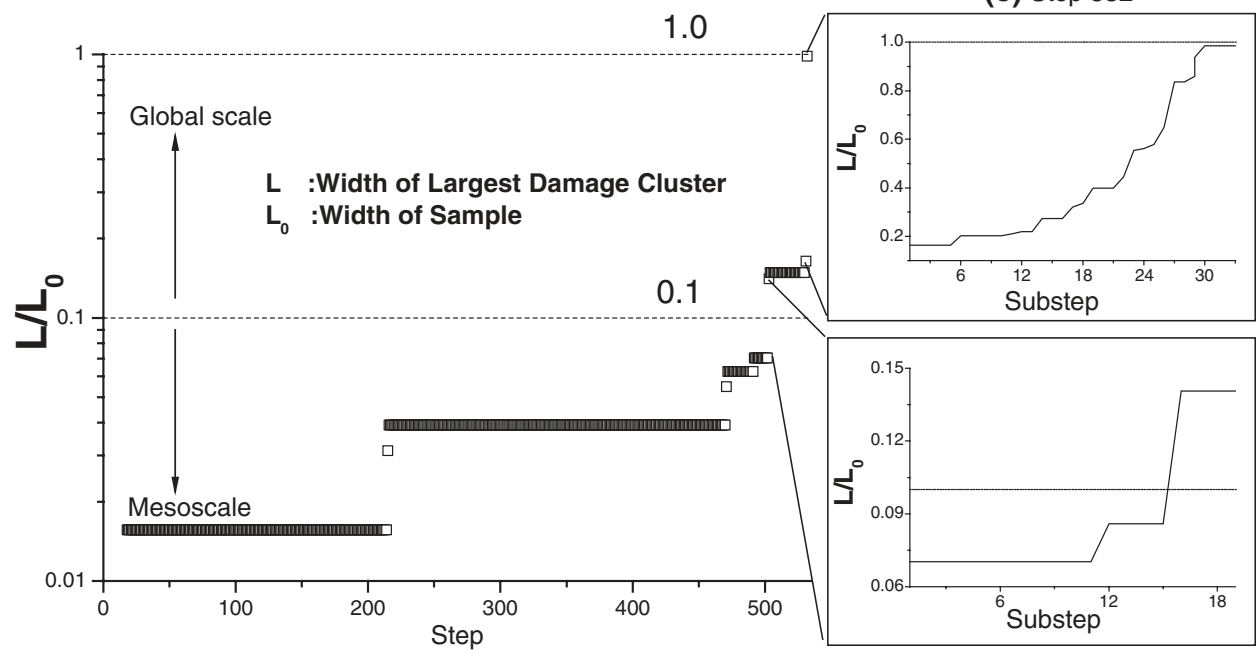

(a)

(b) Step 502

Figure 7

(a) The evolution of the span of the largest damage cluster in the whole damage evolution (from step 0 to step 532) in the plane stress case. (b) The detailed evolution within step 502, and (c) the evolution during eventual catastrophic rupture. The dashed lines stand for the characteristic ratios between the width of the largest damage cluste $(\mathrm{L})$ and the width of the sample $\left(\mathrm{L}_{0}\right)$.

see from Figures 5 and 6 that the damage cluster becomes marginal to the catastrophe transition of the entire specimen. Resultingly the largest damage cluster grows as a trans-scale, inverse cascade of damage from small scale to large scale, and eventually to global scale.

The statistics of the growth of the largest cluster for samples are shown in Figures 9 and 10 for the plane stress and plane strain cases, respectively. Although there is variation in the two different stress states, inverse cascade of damage manifests a common feature of catastrophe transition.

\section{Underlying Mechanism of Catastrophe}

The coalescence of damage which leads to failure is affected by the coupling of nonlinear stress redistribution and heterogeneity. An examination of the evolution of the stress redistribution distance (defined as the distance between newly damaged elements and neighboring elements where the Coulomb stress $\left(\tau_{M C}\right.$ in Equation 2$)$ increases during a specific substep) reveals that the increase in damage results in a concomitant increase in stress and the stress redistribution distance. Figure 11 
(c) Step 649

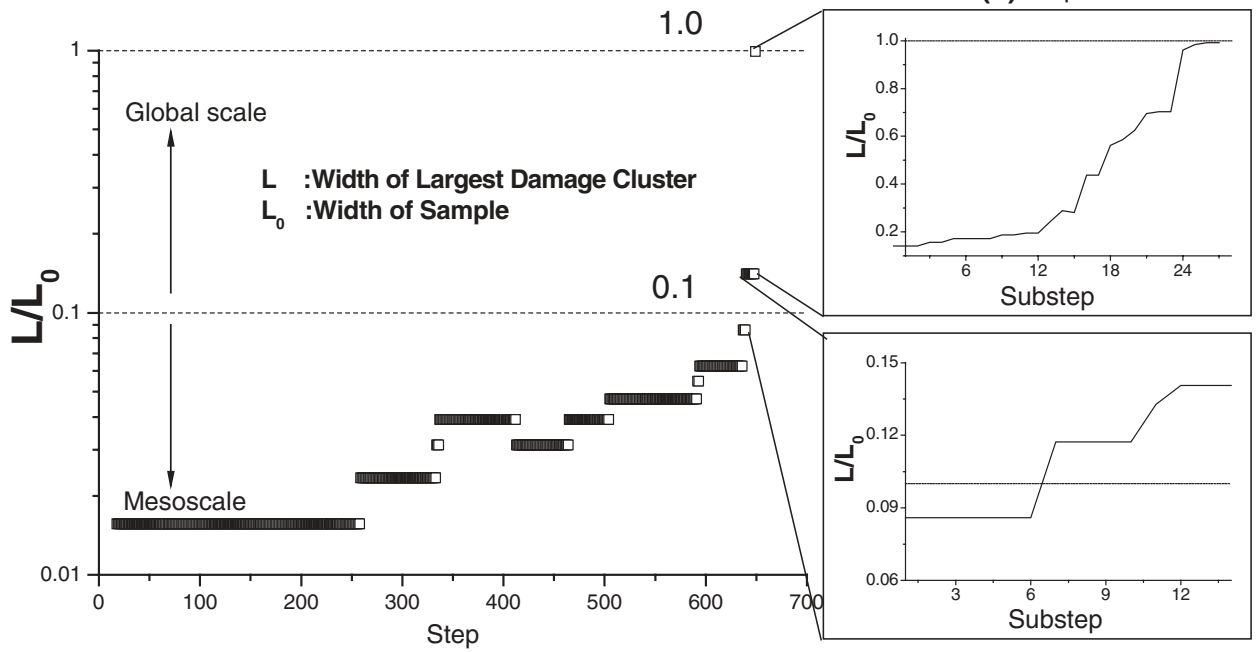

(a)

(b) Step 640

Figure 8

(a) The evolution of the span of the largest damage cluster in the whole damage evolution (from step 0 to step 649) in the plane strain case. (b) The detailed evolution within step 640, and (c) the evolution during eventual catastrophic rupture. The dashed lines stand for the characteristic ratios between the width of the largest damage cluster $(\mathrm{L})$ and the width of the sample $\left(\mathrm{L}_{0}\right)$.

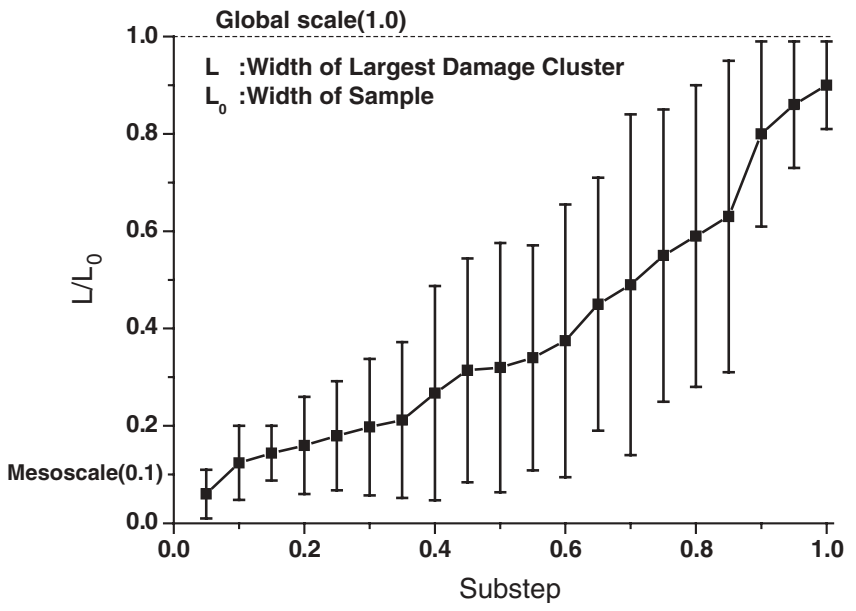

Figure 9

Inverse cascade in the catastrophe transition of the plane stress cases. Fifteen samples were used to calculate the average and the error bars. 


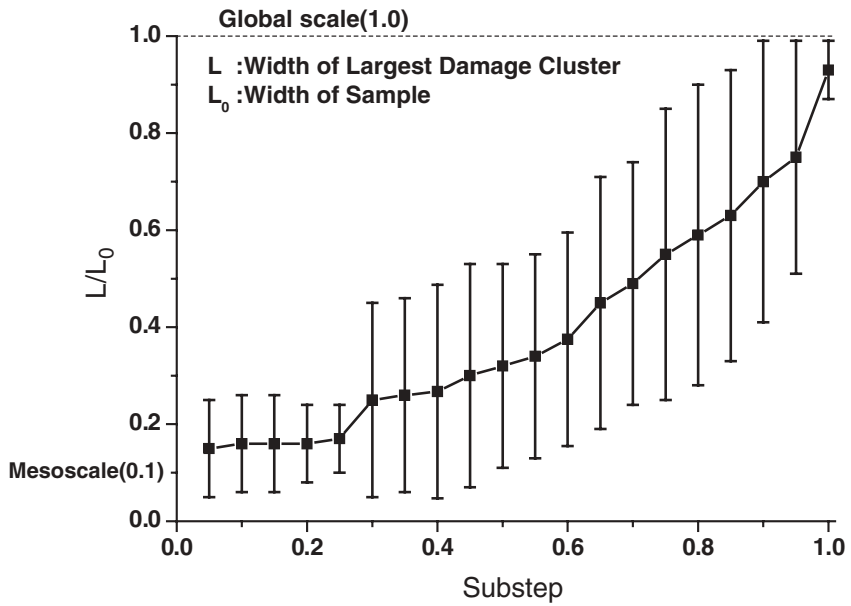

Figure 10

Inverse cascade in the catastrophe transition of the plane strain cases. Fifteen samples were used to calculate the average and the error bars.

shows the stress increase and redistribution distance for the plane stress case. During the early stage of damage accumulation (Fig. 11a), higher stress increment is confined to the elements closer to the damaged elements. As damage accumulates, the stress increment increases and distant elements begin to be affected in addition to those closer to the damaged elements (Fig. 11b). Now, obvious stress redistribution is not only seen near the damaged elements, but also farther away. This phenomenon has a significant effect on the final catastrophic rupture. At the point of catastrophic rupture, elements both proximal and distal to the previously damaged elements experience extreme increases in stress redistribution, indicating complete failure (Fig. 11c). As stress increases, elements with higher strength will be damaged more. This results in a positive feedback mechanism, i.e., stress, damage, and stress redistribution distance become coupled with each other strongly, leading to damage formation at even greater distances. Ultimately, the strong stress fluctuations lead to a breakdown of mean field approximation (ZHANG et al., 2004).

Similar statistics on stress redistribution distance and stress increment in the plane strain case are given in Figure 12. There are also differences in the stress redistribution distance between the plane stress and plane strain cases (Figs. 11 and 12), which make the final damage pattern quite different. Comparing Figures 11(c) and 12(c), the element count reveals that the number of elements where Coulomb stress increases in the plane stain case is greater than that of the plane stress case. And the stress redistribution distance in the plane strain case is lower than that of the plane stress. This statistical result is quite reasonable due to the more 
(a)

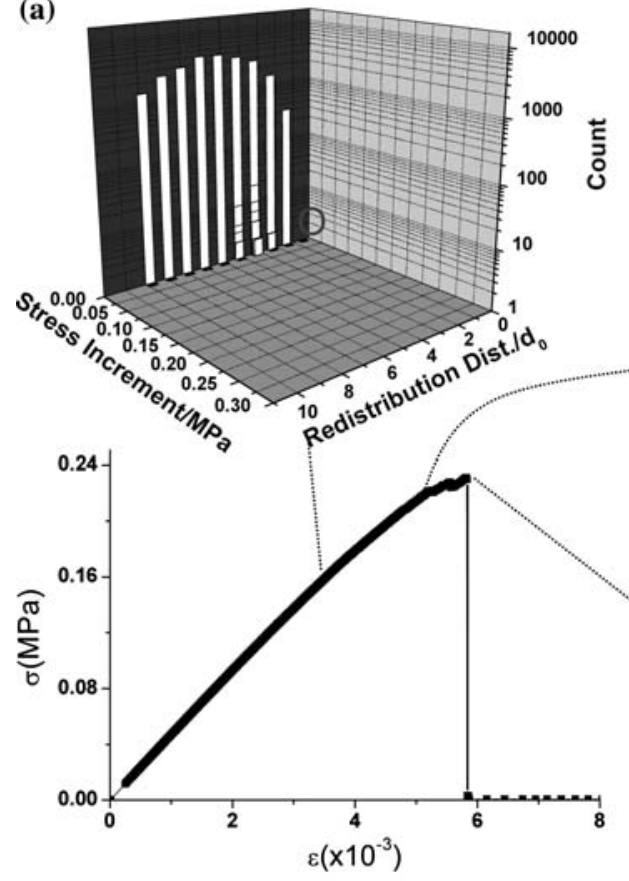

(b)

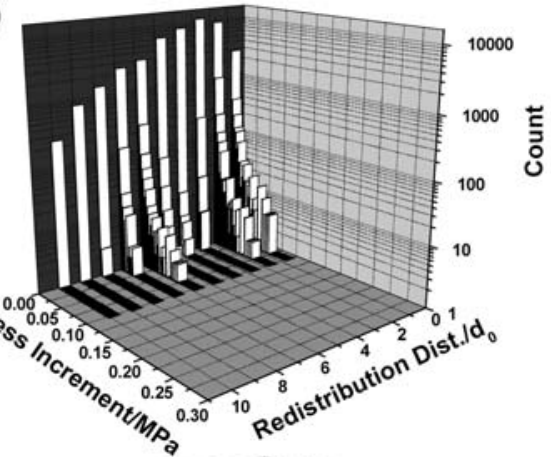

(c)

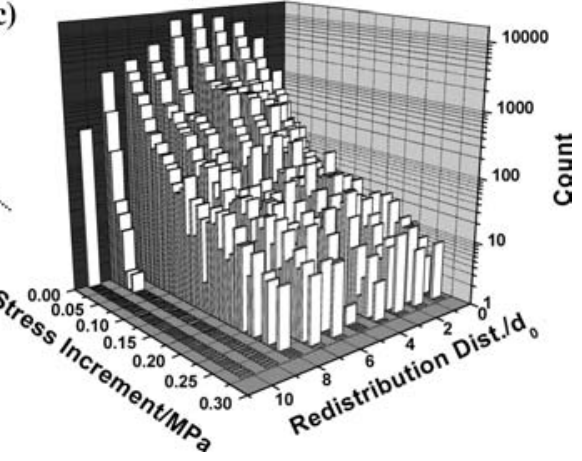

Figure 11

Stress redistribution distance distribution of the plane stress case. Stress increment means the increment of Coulomb stress in elements, redistribution distance indicates the distance between the newly damaged element and the neighboring elements where Coulomb stress increased, and count is the number of elements where the Coulomb stress increased elements. $d_{0}$ is the length of finest elements. (a) The early stage of damage accumulation, (b) the later stage of damage accumulation, (c) the catastrophic rupture.

constrained boundary condition in the plane strain case. The different stress redistribution should be the other reasons, which result in the difference in failure pattern in the two cases.

The coupling of heterogeneity with stress redistribution strongly affects the trans-scale inverse cascade of damage evolution. A comparison of the initial distribution representing the heterogeneity and the counts of the damaged elements at different stages of damage evolution show this coupling effect more clearly (Figs. 13 and 14). In order to clarify this effect even more clearly, let us make a comparison with the mean field approximation. The initial heterogeneity in shear strength is Weibull distribution. Based on mean field approximation (ZHANG et al., 2004), i.e., all elements have the same stress state, all elements with shear strength below the current Coulomb stress will become damaged, see Figure 13(a). However, in the simulations, some elements with higher shear strength are also damaged due to higher stress resulting from the coupling 
(a)

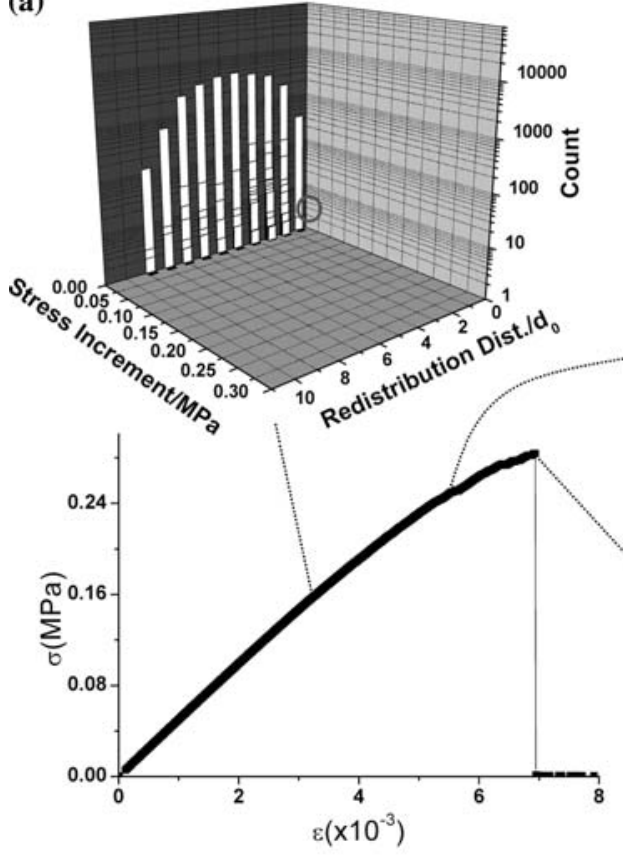

(b)

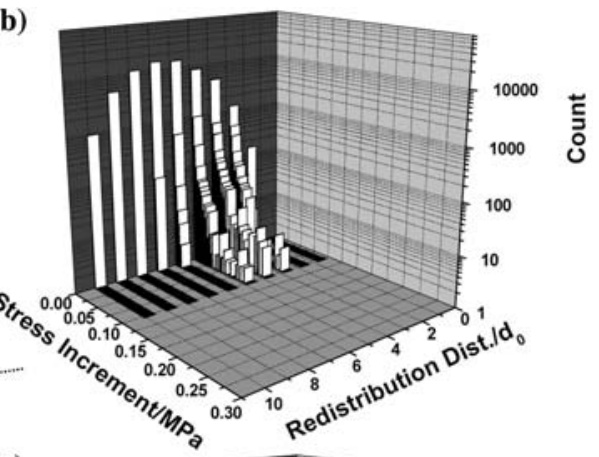

(c)

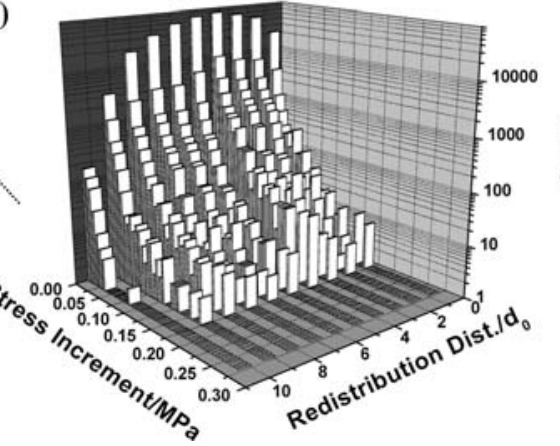

Figure 12

Stress redistribution distance distribution of the plane strain case. $d_{0}$ is the length of finest elements. (a) The early stage of damage accumulation, (b) the later stage of damage accumulation, (c) the catastrophic rupture.

between heterogeneity and stress redistribution, whereas some elements with low shear strength remain intact, as shown in Figures 13(b) and 14(a). Although initial Weibull distribution remains the same in plane stress and strain cases, both final catastrophic patterns (Figs. 3 and 4) and the statistics of damaged elements are different (Figs. 13(c) and 14(b)). All these differ from the global mean field approximation and this clearly demonstrates that the mechanism underlying the catastrophe transition is the coupling between dynamical stress distribution and disordered heterogeneity.

\section{Summary}

We use an adaptive multiscale finite-element model (MFEM) to simulate the damage evolution and the catastrophic failure of heterogeneous brittle media. The linear elastic constitutive relationship is used for intact elements. Damage is introduced with Coulomb failure criterion to each element and the heterogeneity is depicted by Weibull distribution of inherent shear strength in Coulomb criterion and 

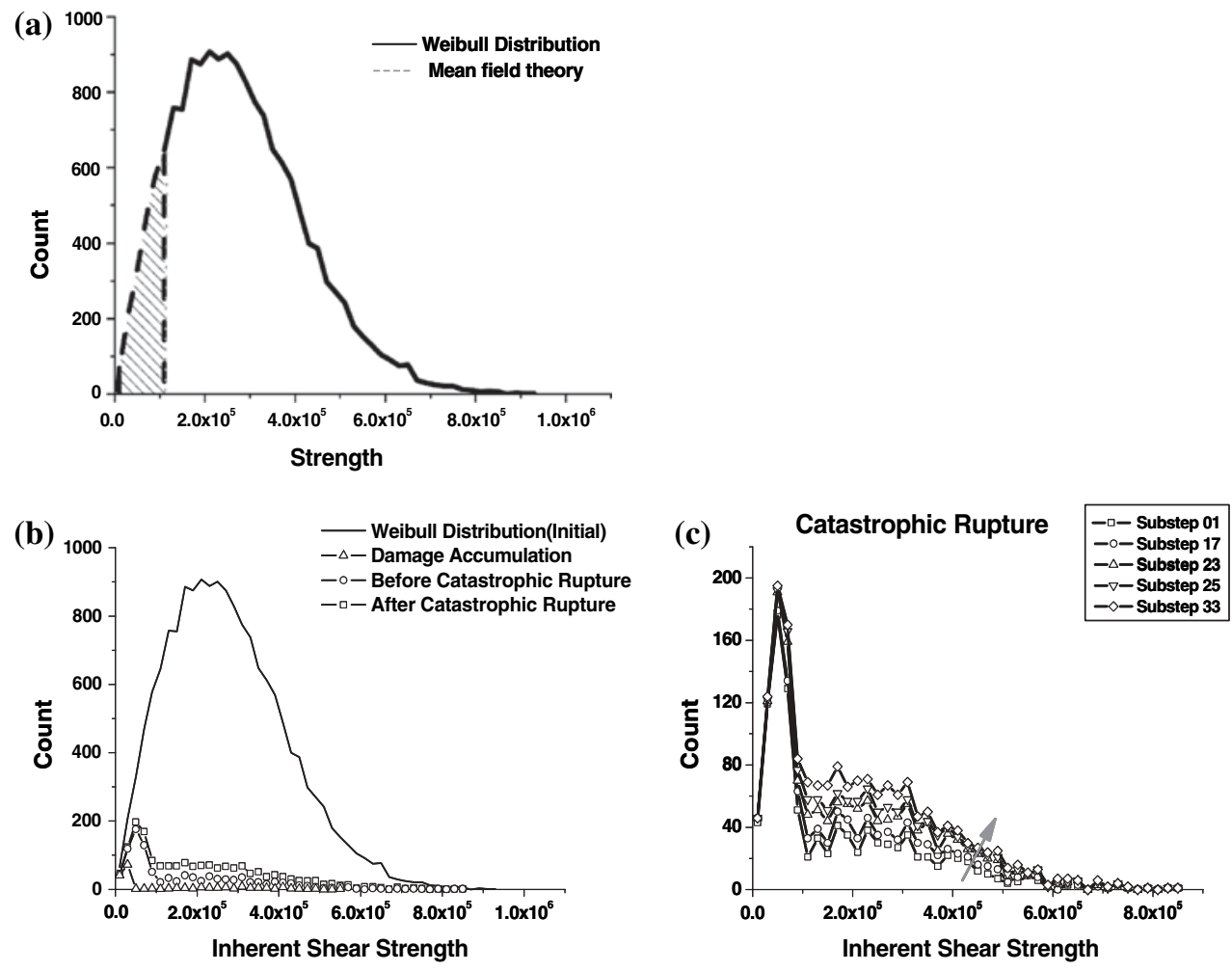

Figure 13

Initial Weibull distribution of shear strength and the counts of damaged elements (the area under corresponding curves) in a plane stress case (a) mean field theory, the area between the Weibull distribution and the vertical dashed line is the total of damaged elements (shaded). (b) The counts for different steps. (c) The counts of zoomed substeps during catastrophic rupture.

random spatial distribution. Large elements should be refined, once the failure criterion is met in them. While the finest elements satisfied the criterion, it will become damaged on three possible orientations where the criterion is satisfied. Consequently, there may be multiple damage in a finest element until complete failure. In order to better understand this catastrophic rupture, a detailed investigation of the damage evolution is carried out, with particular attention to the catastrophe transition. Both plane stress and plane strain cases were investigated. We found that as damage accumulates, there is a strong nonlinear increase in stress and the stress redistribution distance. This is an indication of the mechanism underlying catastrophe transition. The coupling of the dynamic stress redistribution and the material heterogeneity at different scales results in an inverse cascade of damage clusters, which represents the rapid coalescence of damage at catastrophe transition. Therefore, we conclude that the mechanism underlying the catastrophe transition is the coupling between stress redistribution and disordered heterogeneity. 
(a)

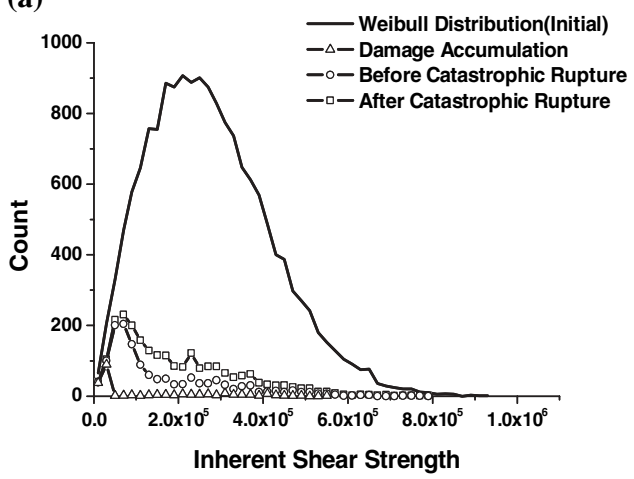

(b)

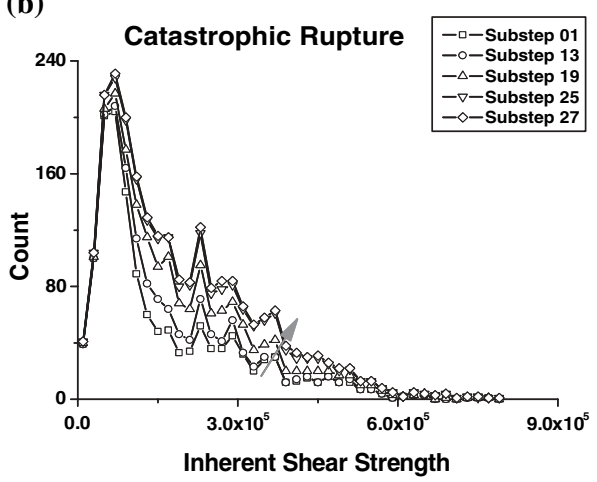

Figure 14

Initial Weibull distribution of shear strength and the counts of damaged elements in the plane strain problem. (a) The counts for different steps. (b) The counts of zoomed substeps during catastrophic rupture.

\section{Acknowledgements}

This research is supported by the National Natural Science Foundation of China (Grant No. 10232050, 10302029, 10472118, 10372012 and 10572139) and the Chinese Academy of Sciences. The authors are grateful for the computation on LSSC-II in State Key Laboratory of Scientific and Engineering Computing, Chinese Academy of Sciences.

\section{REFERENCES}

BAI, Y.L., Lu, C.S., Ke, F.J., and XIA, M.F. (1994), Evolution induced catastrophe, Phys. Lett. A 185, 196-200.

BAZANt, Z.P. and Chen, E.P. (1997), Scaling of structural failure, Appl. Mech. Rev. 50, 593-627.

Ben-Zion, Y. and Sammis, C.G. (2003), Characterization of fault zones, Pure Appl. Geophys. 160, 677-715.

CAO, L.Q., CUI, J.Z., and ZHU, D.C. (2002), Multiscale asymptotic analysis and numerical simulation for the second-order Helmholtz equations with rapidly oscillating coefficients over general convex domains, SIAM J. Numer. Anal. 40 (2), 543-577.

Coleman, B. D. (1958), On the strength of classical fibers and fiber bundles, J. Mech. Phys. Sol. 7, 60-70.

Curtin, W.A. (1997), Toughening in disordered brittle materials, Phys. Rev. B55, 11270-11276.

Daniels, H. E. (1945), The statistical theory of the strength of bundles of threads, Proc. Roy. Soc. London A183, 405-435.

FISH, J. and CHEN, W. (2003), RVE-based multi-level method for periodic heterogeneous media with strong scale mixing. J. Eng. Math. 46, 87-106.

Ghosh, S., Lee, K., and Raghavan, P. (2001), A multi-level computational model for multi-scale damage analysis in composite and porous materials, Int. J. Sol. Struct. 38, 2335-2385.

JAEGER, J.C. and COOK, N.G.W., Fundamentals of Rock Mechanics, 3rd Ed. (Chapman and Hall, London. 1979).

JING, L. and Hudson, J.A. (2002), Numerical methods in rock mechanics, Int. J. Rock Mech. Min. Sci. 39, 409-427. 
Kikuchi, F., Oкаве, M., and Fujio, H. (1999), Modification of the 8-node serendipity element, Comput. Methods Appl. Mech. Engrg. 179, 91-109.

KRAUSE, R. and RANK, E. (2003), Multiscale computations with a combination of the h-and p-versions of the finite-element method, Comput. Methods Appl. Mech. Engrg. 192, 3959-3983.

Mora, P., Wang, Y.C., Yin, C., Place, D., and Yin, X.C. (2002), Simulation of the load-unload response ratio and critical sensitivity in the lattice solid model, Pure Appl. Geophys. 159 (10), 2525-2536.

Paterson, M.S., Experimental Rock Deformation - The Brittle Field (Springer-Verlag, New York 1978).

Rong, F., WAng, H. Y., XIA, M.F., KE, F.J., and BAI, Y.L. (2006), Trans-scale coupling in multi-scale simulation, Int. J. for Multiscale Numer. Engrg. 4, 169-182.

Rong, F., XIA, M.F., KE, F.J., and BAI, Y.L. (2005b), Adaptive mesh refinement FEM for Damage Evolution of Heterogeneous Brittle Media, Model. Simul. Mater. Sci. Engin. 13, 771-782.

Rundle, J.B., Klein, W., Tiampo, K.F., and Gross, S. (2000), Dynamics of seismicity pattern dynamics in systems of earthquake faults, geocomplexity and the physics of earthquakes, AGU Monograph, Washington, D.C., 127-146.

Szabo, B. and Babuska, I., Finite Element Analysis (J. Wiley \& Sons, New York 1991).

Wang, H. Y., He, G. W., XIA, M. F., KE, F. J., and BAI, Y. L. (2004), Multiscale Coupling in complex mechanical systems, Chem. Engin. Sci. 59, 1677-1686.

WeI, Y.J., XIA, M.F., Ke, F. J., YIN, X.C., and BAI, Y. L. (2000), Evolution-induced catastrophe and its predictability, Pure Appl. Geophys. 157, 1945-1957.

XU, X.H., MA, S.P., XIA, M.F., KE, F.J., and BAI, Y. L. (2004), Damage evaluation and damage localization of rock, Theor. Appl. Fract. Mech. 42, 131-138.

XIA, M. F., KE, F. J., and BAI, Y.L. (2000), Evolution-induced catastrophe in a nonlinear dynamical model of materials failure, Nonlin. Dyn. 22, 205-224.

XIA, M.F., WEI, Y.J., KE, F.J., and BAI, Y.L. (2002), Critical sensitivity and trans-scale fluctuations in catastrophic rupture, Pure Appl. Geophys. 159, 2491-2509.

Zhang, X. H., Rong, F., XIA, M.F., Ke, F.J., and BaI, Y.L. (2004), Coupling effects of heterogeneity and stress fluctuation on rupture, Theoreti. Appl. Frac. Mech. 161, 1931-1944.

Zienkiewicz, O.C. and TaYlor, R.L., The Finite-Element Method, 4th ed., vol. 1 (McGraw-Hill, New York 1988).

(Received December 30, 2004, revised November 17, 2005, accepted December 28, 2005)

(12) To access this journal online:

